\title{
Chemical potential and the self-energy correction of spinors: Further results on the large-U Hubbard model
}

\author{
$\mathrm{L} \mathrm{HU}^{\dagger}$ and $\mathrm{K}$ W YU \\ Department of Applied Physics, Hong Kong Polytechnic. Hung Hom, Kowloon, Hong \\ Kong \\ ${ }^{\dagger}$ The Microelectronics Research Institute, Zhongshan University, Guangzhou, People's \\ Republic of China
}

\begin{abstract}
The spinor-holon effective Hamiltonian proposed by Zou and Anderson is used to calculate the chemical potential and the self-energy correction of spinors. taking into account the effect of spin exchange interaction. In the superfluid phase, the transition temperature is sensitive to various choices of the on-site Coulomb repulsion $U$ and the band parameter $t$. The correction due to spin fluctuations is extremely important for small doping fractions $\delta$. In the region of $\delta \leqslant 8 t / \pi^{2} U$, the effect of paramagnon fluctuations renders the mean field theory invalid. The paramagnetic susceptibility $\chi$ in the long wavelength limit is almost independent of temperature $T$ at high temperatures. For fixed $T, \chi$ increases with doping $\delta$ while for fixed $\delta, \chi$ decreases with $T$, in qualitative agreement with experiments.
\end{abstract}

Keywords. Spin fluctuations; quantum spin liquid; Pauli paramagnetism

\section{Introduction}

Since the discovery of high temperature superconductivity (HTSC) in the copper-oxide compounds, a variety of mechanisms have been proposed. Many of the models are based on the two-dimensional Hubbard model for a square lattice of $\mathrm{CuO}_{2}$. The research can be roughly divided into two categories. A notable one is based on the resonating valence bond (RVB) mean field analysis (Baskaran et al 1987; Andrei et al 1987) and the other on the investigation of what role the spin fluctuations played (Bickers 1987; Littlewood et al 1989a, b; Hirsch 1987) in the HTSC mechanism.

In this paper, we discuss the effect of spin fluctuations on the HTSC mechanism (Hu et al 1989a, b, c; Hu and Yu 1989). Starting with the effective Hamiltonian of the large$U$ Hubbard model proposed by Zou and Anderson (1988), we investigate the effect of paramagnon fluctuations on the spinor chemical potential and the self-energy correction.

\section{Spinor self-energy and chemical potential}

We start with the Zou-Anderson large- $U$ effective Hamiltonian in the momentum representation (Zou and Anderson 1988). In the case of large on-site Coulomb potential $U$, after taking averages of the holons, the effective Hamiltonian can be written as

$$
H_{\mathrm{ef} f}=H_{\imath}+H_{J}+\text { constant. }
$$


In (1), the corresponding terms are

$$
\begin{aligned}
& H_{t}=\sum_{\mathbf{k}, \sigma}\left(\varepsilon_{\mathbf{k}, \sigma}-\mu\right) S_{\mathbf{k}, \sigma}^{\dagger} S_{\mathbf{k}, \sigma} \\
& H_{J}=-(Z J / 2 N) \sum_{\mathbf{k}, \mathbf{k}^{\prime}, \mathbf{q}, \sigma} \gamma_{\mathbf{q}}\left[S_{\mathbf{k}+\mathbf{q}, \sigma}^{\dagger} S_{\mathbf{k}^{\prime}-\mathbf{q}, \bar{\sigma}}^{\dagger}\left(S_{\mathbf{k}^{\prime}, \sigma} S_{\mathbf{k}, \sigma}-S_{\mathbf{k}^{\prime}, \sigma} S_{\mathbf{k}, \sigma}\right)\right],
\end{aligned}
$$

where $\varepsilon_{\mathbf{k}, \sigma}=-Z \delta t \gamma_{\mathbf{k}}, \gamma_{\mathbf{k}}=(1 / Z) \Sigma_{z} \exp (i \mathbf{k} \cdot z), Z$ being the number of nearest neighbours denoted by $z(Z=4$ for square lattice $)$ and $J=4 \mathrm{t}^{2} / U . S_{\mathbf{k} \sigma}$ is the spinor operator and $N$ is the total number of lattice sites on the $X Y$ plane. The first term describes the kinetic energy of spinors and the second the exchange interaction. This model has three independent parameters, $t, J$ and the doping fraction $\delta$. The lowest order correction (in $J$ ) for the spinor self-energy can be obtained from the random phase approximation (RPA) calculation (Fetter and Walecka 1971), one gets

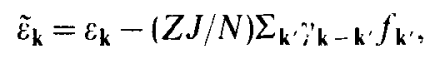

where $f_{k}$ is the Fermi-Dirac distribution at a temperature $T$ and $\beta=1 / k_{B} T$ is the reciprocal temperature and

$$
f_{\mathbf{k}}=\left[1+\exp \beta\left(\tilde{\varepsilon}_{\mathbf{k}}-\mu\right)\right]^{-1} \text {. }
$$

The approximate density of states (DOS) in two dimensions can be written as

$$
\rho(\varepsilon)=\frac{2}{\pi^{2} D} \ln |4 D / \varepsilon|
$$

where $D=4 t \delta$ being the bare half width. By a lengthy but straightforward calculation, one obtains

$$
\tilde{\varepsilon}_{\mathbf{k}}=-\tilde{D} \gamma_{\mathbf{k}}
$$

In the notation of BZA (Baskaran et al 1987; Andrei et al 1987), we can write the renormalized half width as $\tilde{D}=4 \delta t+2 P J$ and

$$
P=\frac{1}{\pi^{2}}\left\{\left[1+4 \ln 2-\left|\frac{\mu}{D}\right|^{2}\left(1+\ln \left|\frac{4 D}{\mu}\right|^{2}\right)\right]+\cdots\right\} .
$$

Since the spinor bare energy is given by $\varepsilon_{\mathrm{k}}=-4 \delta t \gamma_{\mathrm{k}}$, it can be understood that the effect of the spin correlation on the self-energy correction is to enlarge the bare energy band of spinors.

The calculation of the spinor chemical potential is as follows. For large $U$, the relation between the spinor number and the doping fraction can be approximated by $N_{s}=N(1-\delta)=\sum_{\mathbf{k} \sigma} f_{\mathbf{k}}$, hence we get the relation between the chemical potential $\mu$ and the doping fraction $\delta$

$$
\delta=\sum_{\mathbf{k}} \tan h\left[(\beta / 2)\left(\tilde{\varepsilon}_{\mathbf{k}}-\mu\right)\right] .
$$

Here for consistency, we use the renormalized spinor energy $\tilde{\varepsilon}_{\mathbf{k}}$. We obtain the following expression

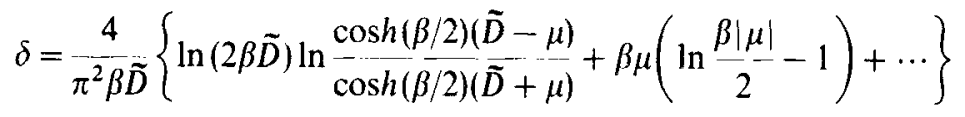


giving the doping fraction $\delta$ as a function of $\mu, \tilde{D}$ and $T$. The numerical results show that for low temperatures and fixed $\delta$, with $t=0.2$ to $0.5 \mathrm{eV}$ and $U=2$ to $5 \mathrm{eV}$, the chemical potential $\mu$ and the renormalized spinor half bandwidth $\tilde{D}$ are nearly independent of temperature, but both change rapidly with the doping fraction $\delta$. The larger $t$ becomes, the faster $\mu$ decreases with increasing $\delta$. but $\mu$ remains nearly zero in the region $\delta<0.05$, with $\mu=0$ when $\delta=0$, which satisfies the half-filling condition. Moreover, the correction of spin fluctuations is extremely important for small $\delta$. For $t=0.05 \mathrm{eV}$ and $U=2.5 \mathrm{eV}$, the correction doubles the bare half-width when $\delta \approx 0 \cdot 15$, thus rendering the RPA invalid.

\section{Spinor superfluid phase}

From equation (1) and by using the renormalized bandwidth and DOS, we obtain the following integral equation which determines the $S$-wave superfluid transition temperature $T_{s}$.

$$
\frac{\pi^{2} \tilde{D}}{8 J}=\int_{0}^{1} x^{2} \ln (4 / x)\left(\frac{\tanh \left[\beta_{c} \tilde{D}(x+y)\right]}{x+y}+\frac{\tanh \left[\beta_{c} \tilde{D}(x-y)\right]}{x-y}\right) \mathrm{d} x,
$$

where $y=\mu / \tilde{D}$ and $\beta_{c}=1 / k_{B} T_{s}$. Taking $t=0 \cdot 5 \mathrm{eV}$ and $t / U=0 \cdot 2$, and by numerical calculations, we obtain the relation between the $S$-wave transition temperature $T_{s}$ and the doping fraction $\delta$. The effect of increasing the doping fraction or increasing $U$ is to increase the relative kinetic energy of spinors, leading to the decrease of the superfluid transition temperature $T_{s}(\delta)$, which is sensitive to different choices of the parameters $t$ and $U$. The increase of $U$ tends to shift the boundary of $T_{s}(\delta)$ to the left. While our results agree qualitatively with those of BZA (Baskaran et al 1987; Andrei et al 1987) at large doping fraction, the discrepancy becomes significant for small doping fractions, indicating that the correction due to spin fluctuations cannot be ignored for small $\delta$.

\section{Pauli paramagnetism}

In the following, we present the calculations of the Pauli susceptibility. From the RPA calculations,

$$
\chi(\mathbf{q})=\chi_{0}(\mathbf{q}) /\left[1+\tilde{J}(\mathbf{q}) \chi_{0}(\mathbf{q})\right]
$$

where

$$
\tilde{J}(\mathbf{q})=4 Z J \gamma_{\mathbf{q}} / g_{L}^{2} \mu_{B}^{2}
$$

and

$$
\chi_{0}(\mathbf{q})=\frac{g_{L}^{2} \mu_{B}^{2}}{4 N} \sum_{\mathbf{k}} \frac{f_{\mathbf{k}}-f_{\mathbf{k}-\mathbf{q}}}{\bar{\varepsilon}_{\mathbf{k}-\mathbf{q}}-\check{\varepsilon}_{\mathrm{k}}}
$$

where $g_{L}$ and $\mu_{B}$ being the Lande factor and the Bohr magneton respectively. We use the renormalized density of states to calculate the susceptibility in the long wavelength limit. Taking $\mathbf{q}=0$ in equations (11) - (13), we find that $\chi$ is almost independent of 
temperature $T$ at high temperatures. For fixed $T, \chi$ increases with the doping $\delta$ while for fixed $\delta, \chi$ decreases with $T$, in qualitative agreement with experiment (Ishii et al 1987). The increase of $\chi(\delta, T)$ with $\delta$ at fixed $T$ can be explained by the increase of density of states on the spinor pseudo Fermi surface with the doping fraction.

\section{Conclusions}

We have used the spinor-holon effective Hamiltonian proposed by Zou and Anderson to calculate the chemical potential and the self-energy correction of spinors. Our results show that the effect of spin exchange interaction is to enlarge the spinor bandwidth. In the superfluid phase, the effect of increasing the doping fraction or increasing the onsite Coulomb potential is to increase the relative kinetic energy of spinors, leading to the decrease of superfluid transition temperature, which is sensitive to various choices of $U$ and the band parameter $t$. The increase of $U$ tends to shift the boundary of $T_{s}(\delta)$ towards lower doping fraction. The correction due to spin fluctuations is extremely important for small doping fractions, leading to the modification of the results of Baskaran et al (Baskaran et al 1987; Andrei et al 1987). Moreover, in the region of $\delta \leqslant 8 t / \pi^{2} U$, the effect of paramagnon fluctuations renders the mean field theory invalid. We also calculated the paramagnetic susceptibility $\chi$ in the long wavelength limit. We find that $\chi$ is almost independent of temperature $T$ at high temperatures. For fixed $T, \chi$ increases with doping $\delta$ while for fixed $\delta, \chi$ decreases with $T$, in qualitative agreement with experiments. The present analysis is more convenient to study the magnetic properties of the two-dimensional large- $U$ Hubbard model.

\section{References}

Andrei E R. Peter J H and Appel J 1987 Phys. Rev. B36 857

Baskaran G, Zou Z and Anderson P W 1987 Solid State Commun. 68973

Bickers N E 1987 Int. J. Mod. Phys. B1 687

Fetter A L and Walecka J D 1971 in Quantum theory of many-particle systems (New York: McGraw Hill) p. 227

Hirsch J E 1987 Phys. Rev. B35 8726

Hu L and Yu K W 1989 (Unpublished)

Hu L, Wu S S and Hua X M 1989a Acta Sci. Nat. Uni. Sunyatseni 3105

Hu L, Shi L P and Hua X M 1989b Phys. Rev. B40 11306

Ishii H et al 1987 Physica B148 419

Littlewood P B et al 1989a Phys. Rev. B39 12371: 1989b Preprint

Zou Z and Anderson P W 1988 Phys. Rev. B37 627 